\title{
Free Energies of the Disassembly of Viral Capsids from a Multiscale Molecular Simulation Approach
}

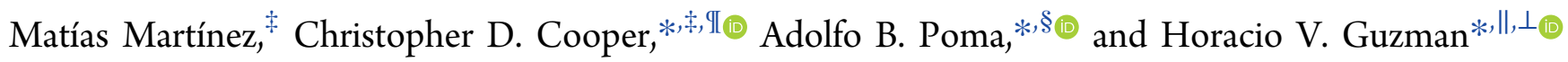 \\ ${ }^{\ddagger}$ Department of Mechanical Engineering, Universidad Técnica Federico Santa María, 2390123 Valparaíso, Chile \\ ${ }^{\text {II }}$ Centro Científico Tecnológico de Valparaíso (CCTVal), 2390123 Valparaíso, Chile \\ ${ }^{\S}$ Institute of Fundamental Technological Research, Polish Academy of Sciences, Pawińskiego 5B, 02-106 Warsaw, Poland \\ "Max Planck Institute for Polymer Research, Ackermannweg 10, 55128 Mainz, Germany \\ ${ }^{\perp}$ Jožef Stefan Institute, Jamova 39, 1000 Ljubljana, Slovenia
}

Supporting Information

\begin{abstract}
Molecular simulations of large biological systems, such as viral capsids, remains a challenging task in soft matter research. On one hand, coarse-grained (CG) models attempt to make the description of the entire viral capsid disassembly feasible. On the other hand, the permanent development of novel molecular dynamics (MD) simulation approaches, like enhanced sampling methods, attempt to overcome the large time scales required for such simulations. Those methods have a potential for delivering molecular structures and properties of biological systems. Nonetheless, exploring the process on how a viral capsid disassembles by all-atom MD simulations has been rarely attempted. Here, we propose a methodology to analyze the disassembly process of viral capsids from a free energy perspective, through an efficient combination of dynamics using coarse-grained models and Poisson-Boltzmann simulations. In

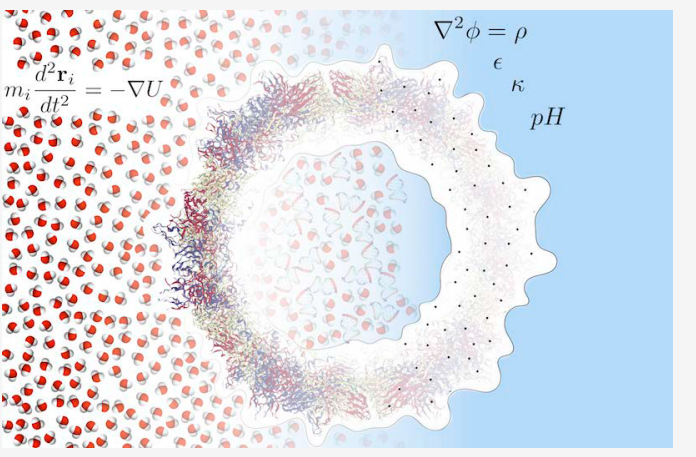
particular, we look at the effect of $\mathrm{pH}$ and charge of the genetic material inside the capsid, and compute the free energy of a disassembly trajectory precalculated using CG simulations with the SIRAH force field. We used our multiscale approach on the Triatoma virus $(\mathrm{TrV})$ as a test case, and find that even though an alkaline environment enhances the stability of the capsid, the resulting deprotonation of the genetic material generates a Coulomb-type electrostatic repulsion that triggers disassembly.
\end{abstract}

\section{INTRODUCTION}

Numerous virus structures are comprised of smaller proteincoat pieces which form a capsid. Such macromolecular structures have, at least, two crucial roles to play, namely: the packaging of the viral genome and the transport of such genetic material throughout the human body. The latter role makes the capsid an efficient and natural nanocarrier, as identified by scientists and the biotechnology industry, for the sake of incorporating those transport abilities to the development of drug delivery applications. Nonetheless, the understanding of how this efficient viral capsids transport works, is still a subject of research from both theoretical and experimental viewpoints. ${ }^{1-6}$ Among those research topics, one question we are interested in is the release of the enclosed material carried by a viral capsid.

Different molecular simulation strategies with special focus on biological processes have been developed during the last 2 decades, those methodologies are designed to describe large conformational changes of biomolecules at the level of the single structure of proteins or their assemblies under natural environments. ${ }^{7,8}$ Such structural changes can occur spontaneously, typically triggered by thermodynamic factors (e.g., $\mathrm{pH}$, temperature, ionic concentrations, etc.) or through local forces which disrupts the mechanical stability of the complex. Viral capsid disassembly ${ }^{9}$ involves the dissociation of protein-coats either for the release of the nucleic acid material during the viral replication process or to regulate protein dissociation by the ionic concentration. In this regard, coarse-grained models ${ }^{10}$ have been derived from thermodynamic principles, e.g., the SIRAH, ${ }^{11,12}$ PRIMO, ${ }^{13}$ MARTINI, ${ }^{14,15}$ and UNRES force fields. ${ }^{16}$ Primarily, those models are designed to allow the description of biological systems by taking into account the typical length and time scales associated with molecular processes in biology.

Here, we focus on $\mathrm{pH}$ gradients between the inside (interior) and outside of the viral capsid. To this end, we propose a novel computational method to estimate the electrostatic free energy values caused by the $\mathrm{pH}$ gradients along viral capsids. This method uses a Poisson-Boltzmann (PB) model for free energy calculations on a detailed structure of the protein-coats disassembly stages, obtained previously by

Special Issue: Molecular Simulation in Latin America: Coming of Age

Received: September 30, 2019

Published: December 24, 2019 


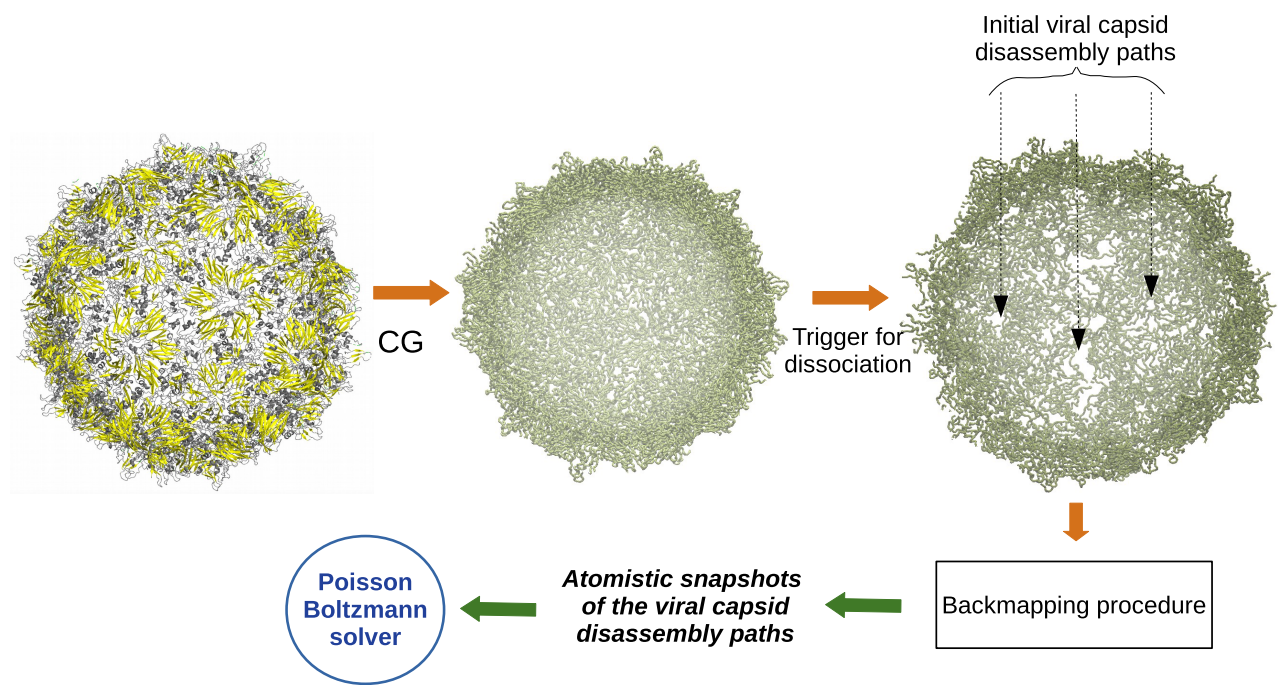

Figure 1. Step-wise scheme of the disassembly process simulation of a $\operatorname{TrV}$ virus via multiscale methods, obtaining the molecular trajectories of such process, and the conversion of scales to fit the Poisson-Boltzmann atomistic configuration requirements for quantification. Note that for visualization reasons the solvent has not been illustrated within this scheme.

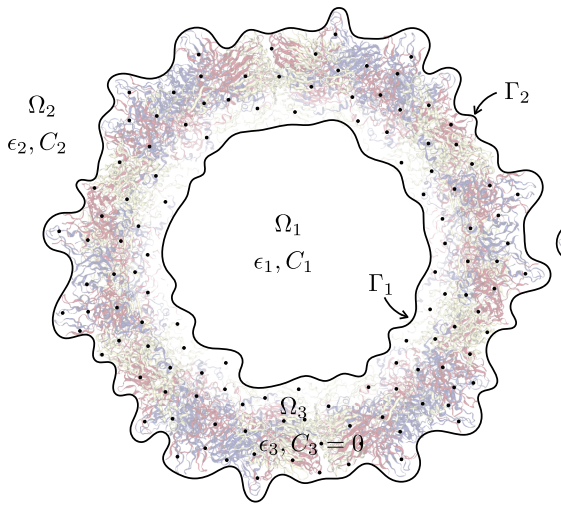

(a)

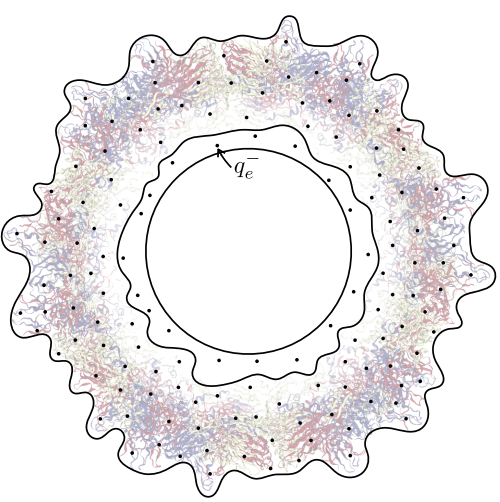

(b)

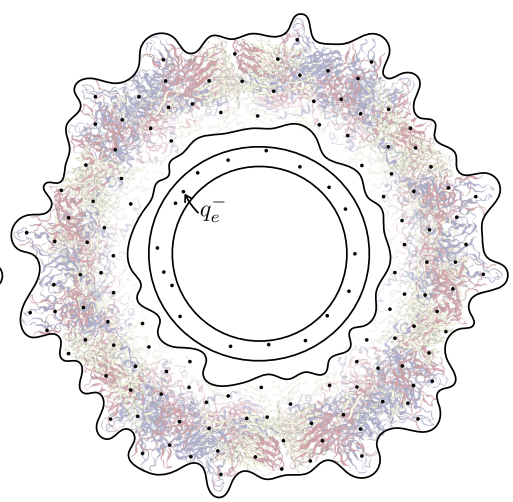

(c)

Figure 2. Representation of the capsid in an implicit-solvent model which comprises three regions (a): inside the capsid $\left(\Omega_{1}\right)$, outside the capsid $\left(\Omega_{2}\right)$ and the interface region where the proteins of the capsid are located $\left(\Omega_{3}\right)$. Part b shows an alternative representation adding an extra region with point charges to represent the charge after the deprotonation of genetic material. After disassembly, the capsid is not watertight, and we place the ions in a spherical shell like in part c. The thin region with negative point charges has the dielectric constant of the solvent, but no salt.

multiscale simulations ${ }^{17-24}$ employing the SIRAH forcefield. ${ }^{11,12}$ This allows us to study the free energy landscapes of those disassembly snapshots and determine which of the configurations is energetically more favorable. On this subject, we aim to pave the way for extending theoretical virology models and also interpreting experiments that attempt to understand the disassembly process of viral and viral-like capsids.

In order to show the quantitative capabilities of our computational method, we have selected a triatoma virus capsid. $^{25}$ This nonenvelope, ssRNA virus is the main vector of the Chagas disease, and is considered to have great potential in the drug development field, according to the World Health Organization. $^{26}$

\section{METHODS}

SIRAH Method to Capture the Conformational Changes of TrV Capsid Disassembly. To obtain atomistic snapshots from the viral capsids disassembly path, we used a backmapping procedure of CG simulations with the SIRAH force field, ${ }^{11,12}$ following previously reported simulations of the initial disassembly paths of $\mathrm{TrV}$ capsids. ${ }^{17}$ In particular, Viso et al. ${ }^{17}$ explored the mechanisms which build the protonation channels across the $\operatorname{TrV}$ capsid, triggering disassembly. The CG ensembles representing the $\mathrm{TrV}$ capsid follow the SIRAH force field, as well as the solvent which uses a multiscale approach based on layers of CG water (WT4 and WLS) as previously reported by Machado et al. ${ }^{27}$ The system equilibration stage was run for around $2 \mu$ s using GROMACS version $2018,{ }^{28,29}$ while the initial disassembly paths where obtained by means of an electrostatic repulsion effect. In other words, CG water molecules have been replaced by ions of $\mathrm{Cl}^{-}$ inside the capsid and simulated them for 500 ps, which generated the required trajectories to observe initial disassemble paths on the TrV. Note here, that the electrostatic repulsion effects caused by the $\mathrm{Cl}^{-}$ions decay rapidly from a "disassembly peak" within the first 200 ps of simulation time, detailed information about such behavior has been reported elsewhere. $^{17}$ Afterward, we employed SIRAH-tools ${ }^{30}$ for backmapping the CG capsids representations to all-atom 
snapshots. The latter is used as an input to our PoissonBoltzmann method to study the solvation energy landscapes of such disassembly process (as illustrated in Figure 1). It is important to remark that the atomistic resolution snapshots are given as an input to our method can be also provided from allatom simulations, or the use of other multiscale methods, like enhanced sampling e.g. TAMD ${ }^{31}$ or new emerging highresolution imaging techniques. $1,32,33$

Regarding performance, the $\operatorname{TrV}$ system $\left(V_{T}=50.41 \times\right.$ $\left.47.53 \times 41.16 \mathrm{~nm}^{3}\right)$ is comprised by roughly $380 \mathrm{k}$ atoms in the capsid, 32k WT4 (approximately $352 \mathrm{k}$ water molecules) and 42k WLS (approximately 2.3 M water molecules) CG beads of solvent which took $15.5 \mathrm{~ns}$ per day by using 2 GPUs Quadro rtx5000. In addition, all the configuration files used in our method are provided in ref 34 under Creative Commons license 4.0.

Poisson-Boltzmann Solver. Rather than considering water molecules explicitly, an implicit solvent model averages their influence in a continuum dielectric description. ${ }^{35,36}$ Then, a dissolved molecule is represented as a multidielectric infinite domain with, at least, two regions-the solute and the solvent-interfaced by the solvent-excluded surface (SES). Figure 2a sketches this situation in the case of a capsid, which has three distinct regions:

- internal $\left(\Omega_{1}\right)$, which is usually filled with genetic material;

- external $\left(\Omega_{2}\right)$, representing the solvent; and

- capsid $\left(\Omega_{3}\right)$, the proteic membrane that interfaces the internal and external regions.

Each region is characterized by its dielectric constant $\left(\epsilon_{1}, \epsilon_{2}\right.$, $\left.\epsilon_{3}\right)$, and salt concentration $\left(C_{1}, C_{2}, C_{3}\right)$, which is zero in the capsid. For simplicity, we will use parameters that are proper for a solvent in the internal region $\left(\Omega_{1}\right)$. If the capsid is not watertight, $\Omega_{1}$ and $\Omega_{2}$ would be connected and considered to be the same dielectric region.

The partial charges in the solute are represented as static point charges at the locations of the atoms, whereas the salt ions in the solvent are considered as mobile point charges that arrange according to Boltzmann statistics. Applying continuum electrostatic theory on this arrangement gives rise to a system of coupled partial differential equations, where the potential inside the solute is governed by Poisson, and in the solvent by Poisson-Boltzmann:

$$
\begin{aligned}
& \left(\nabla^{2}-\kappa_{1}{ }^{2}\right) \phi_{1}=0 \text { on } \Omega_{1} \\
& \left(\nabla^{2}-\kappa_{2}{ }^{2}\right) \phi_{2}=0 \text { on } \Omega_{2} \\
& \nabla^{2} \phi_{3}=\frac{1}{\epsilon_{3}} \sum_{k=1}^{N_{q}} q_{k} \delta\left(\mathbf{r}_{k}\right) \text { on } \Omega_{3} \\
& \phi_{1}=\phi_{3} \text { and } \epsilon_{1} \frac{\partial \phi_{1}}{\partial \mathbf{n}}=\epsilon_{3} \frac{\partial \phi_{3}}{\partial \mathbf{n}} \text { at } \Gamma_{1} \\
& \phi_{2}=\phi_{3} \text { and } \epsilon_{2} \frac{\partial \phi_{2}}{\partial \mathbf{n}}=\epsilon_{3} \frac{\partial \phi_{3}}{\partial \mathbf{n}} \text { at } \Gamma_{2}
\end{aligned}
$$

Here, $\phi$ is the electrostatic potential, $\kappa$ is the inverse of the Debye length (which depends on the salt concentration $C$ ), $\epsilon$ is the dielectric constant, and $q$ is the partial charges of the biomolecule. $\Gamma_{1}$ and $\Gamma_{2}$ are the SES on the inside and outside of the capsid, interfacing $\Omega_{3}$ with $\Omega_{1}$ and $\Omega_{2}$, respectively. The unit vector $\mathbf{n}$ is normal to the SES, and points away from the region enclosed by the surface.

The most common quantity of interest is the solvation energy, which is the work required to bring the solute from vacuum into the solvent. Considering that the charges inside the solute are Dirac delta functions, this energy becomes

$$
\Delta G_{\text {solv }}=\int_{\Omega_{3}} \rho \phi_{\text {reac }}=\sum_{k=1}^{N_{q}} q_{k} \phi_{\text {reac }}\left(\mathbf{r}_{k}\right)
$$

where $\phi_{\text {reac }}=\phi_{3}-\phi_{\text {coul }}$ is the reaction potential, $\rho$ the charge distribution, and $\mathbf{r}_{k}$ the location of charge $k$.

There is another source of energy in the point-charge distribution of the partial charges in the solute. Then, the total electrostatic contribution to free energy is the sum of the solvation energy in eq 2 and the Coulomb-type energy

$$
G_{\text {elec }}=\Delta G_{\text {solv }}+G_{\text {coul }}
$$

where

$$
G_{\text {coul }}=\frac{1}{2 \epsilon_{3}} \sum_{k=1}^{N_{q}} \sum_{j=1}^{N_{q}} q_{k} \frac{q_{j}}{4 \pi\left|\mathbf{r}_{k}-\mathbf{r}_{j}\right|}
$$

The free energy also has a nonelectrostatic component, ${ }^{36,37}$ which accounts for the work required to create the cavity in the solvent that will hold the charges of the solute. The disassembly process is governed by electrostatic interactions; ${ }^{17}$ hence, the nonpolar component of the free energy is negligible, and we only focus on the electrostatic energy.

In this work, we use the Poisson-Boltzmann solver $\mathrm{PyGBe}^{38,39}$ to compute the electrostatic potential and energy in eqs 1 and 3. PyGBe formulates the system of partial differential equations in a boundary integral form, requiring to mesh the SES only, and solves the resulting system with a boundary element method (BEM). The code is memory efficient and fast, ${ }^{40}$ as it uses acceleration from a treecode algorithm, ${ }^{41,42}$ and runs on GPUs. Further details on PyGBe and its implementation can be found elsewhere. ${ }^{38}$ In this work, we developed a shared-memory parallel CPU implementation of PyGbe to be able to simulate large virus-like particles. The PyGBe code is an open source project available on Github, ${ }^{43}$ and is released under a BSD 3 Clause license.

There are important simplifications involved with the Poisson-Boltzmann model; for example, it neglects steric effects and considers a rigid solute. However, the model is still accurate ${ }^{35,36,44}$ and widely used in the biophysics community. ${ }^{30,45,46}$ Moreover, it is a specially useful tool for solvation energy calculations, which are difficult and time-consuming with molecular dynamics.

Preparation of the Capsid for the Electrostatic Calculations. We obtained the molecular structure of the $\mathrm{TrV}$ capsid from molecular dynamics simulations using SIRAH, reparameterized it with the pdb2pqr software ${ }^{51}$ using an amber force field, and meshed the SES with the Nanoshaper software. ${ }^{47}$ We further manipulated the mesh with the Python library trimesh, to remove triangulations on small pockets. This yielded a system like the one shown in Figure 2a, where the capsid membrane $\left(\Omega_{2}\right)$ separates an outside $\left(\Omega_{3}\right)$ and an inside region $\left(\Omega_{1}\right)$. In reality, the inside region contains the genetic material of the virus; however, as a first approach, we will model it as being filled with solvent. Our PoissonBoltzmann approximation computes free energies directly, ${ }^{35,36}$ which includes the entropic and internal energy components 
implicitly. Nevertheless, there are more elaborate models that consider structural details of the nucleic acid explicitly, ${ }^{48-50}$ which could be coupled to a Poisson-Boltzmann solver.

To simulate a $\mathrm{pH}$ difference between the interior and exterior regions, we protonated the $\mathrm{TrV}$ structure using the pdb2pqr software, ${ }^{51}$ depending on the location of each residue. If the residue's $\alpha$ carbon was closer to the surface interfacing with the exterior solvent, the residue was protonated according to the exterior $\mathrm{pH}$, whereas if the $\alpha$ carbon was closer to the interior surface, the hydrogen atoms were assigned with the interior $\mathrm{pH}$.

\section{RESULTS AND DISCUSSION}

The study of the mechanism of virus disassembly is a real challenge for current all-atom MD simulation. On the first hand, it involves the simulation of several millions of particles, and second, the time scales are beyond the standard for MD simulations. In order to overcome those limitations and provide molecular structures of capsids under a disassembly process for further energy landscapes analysis via our PBsolver, we explored a pioneering work ${ }^{17}$ on viral capsids initial disassembly of the $\operatorname{TrV}$. There, the authors employ multiscale simulations to obtain a structural changes on the viral capsids triggered by a Grotthuss-like mechanism for proton channeling, which is used as a hypothesis on how to simulate the electrostatic repulsion inside the capsid and hence deliver CG structures of the capsids under disassembly. We adopted this approach $^{17}$ to compute the electrostatic component of the free energy for molecular structures obtained from multiscale simulations using SIRAH (as illustrated in Figure 1). Those simulations consider a solvent $\left(\Omega_{1}\right.$ and $\Omega_{2}$ in Figure $\left.2 \mathrm{a}\right)$ with $150 \mathrm{mM}$ of salt $\left(\kappa=0.125 \AA^{-1}\right)$ and permittivity 80 . On the other hand, the proteic region $\left(\Omega_{3}\right.$ in Figure $\left.2 \mathrm{a}\right)$ has permittivity 4 .

Furthermore, we bring to discussion for the computational biophysics community an alternative methodology for the calculation of the disassembly pathways. This indicates the possibility to combine within our approach other enhanced sampling methods ${ }^{52,53}$ based on the collective variables such as the temperature accelerated molecular dynamics (TAMD) approach. $^{54}$

The input files required to reproduce the results in this section with the PyGBe code are available on Zenodo, ${ }^{34}$ and shared under Creative Commons Attribution 4.0 International License.

Mesh refinement study. To find an appropriate mesh density, we performed simulations at neutral $\mathrm{pH}$ with 3.16 , 4.88 , and 7.42 boundary elements per $\AA^{2}$, and used Richardson extrapolation ${ }^{38}$ to estimate the solvation energy at infinite refinement. These results are shown in Table 1 , and yield an extrapolated value of $-627,042 \mathrm{kcal} / \mathrm{mol}$.

The solvation energy computed with 3.16 elements per $\AA^{2}$ is $0.4 \%$ away from the infinitely refined solution. This accuracy is appropriate in our case, where solvation energy differences are

Table 1. Solvation Energies Using Different Mesh Refinements for the TrV Capsid at $\mathrm{pH}=7$

$\begin{array}{ccc}\text { density }\left(\mathrm{el} / \AA^{2}\right) & \text { no. of elements } & \Delta G_{\text {solv }}(\mathrm{kcal} / \mathrm{mol}) \\ 3.16 & 2774684 & -629760 \\ 4.88 & 4373808 & -628326 \\ 7.42 & 6761872 & -627648\end{array}$

beyond $0.4 \%$; hence, we use this mesh density in the rest of our simulations. With this mesh size, each simulation took $\sim 4$ $\mathrm{h}$ to complete on a 24-core workstation with 96GB of RAM.

Influence of the $\mathrm{pH}$ Gradient on the Free Energy. We performed calculations of the electrostatic component of free energy on the viral capsid assembled structure (PDB ID: 3NAP) with different values of $\mathrm{pH}$ in the inside and outside regions. Following the disassembling mechanism proposed by Viso and co-workers, ${ }^{17}$ we studied $\mathrm{pH}$ variations between 7 and 8.5. Note that those values are comprised in the ranges used in the literature. ${ }^{55,56}$ Moreover, the drug development community for the experimental characterization of virus-like particles (VLPs) uses also ranges of $\mathrm{pH}$ between 6 and 9 for their vaccines development. ${ }^{57}$ Initially we varied the external $\mathrm{pH}$ maintaining a fixed neutral $\mathrm{pH}$ inside, to then modify the internal $\mathrm{pH}$ with $\mathrm{pH}=8.5$ externally. These results are shown in Figure 3, Figure 4, and Table S1 of the Supporting Information.

Usually, in Coulomb energy calculations, force fields include scaling factors for atoms that are 3 bonds away or less. We did not consider these in the computations that led to Tables 2

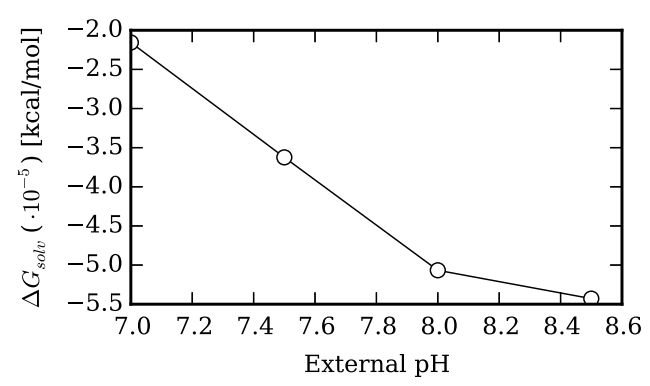

(a) Solvation energy

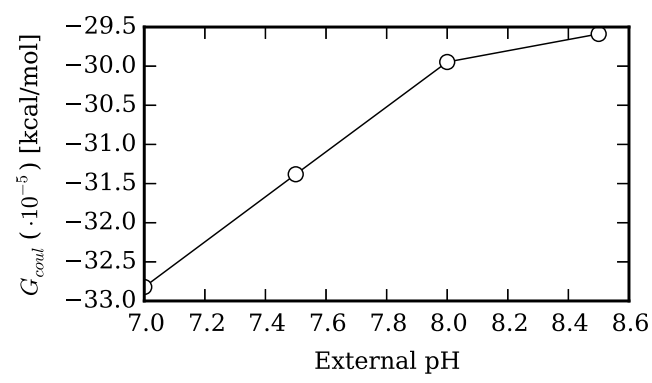

(b) Coulomb energy

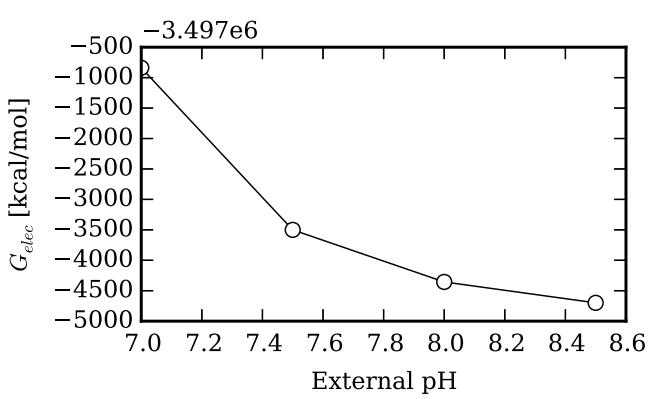

(c) Total electrostatic energy

Figure 3. Electrostatic free energy with varying external $\mathrm{pH}$ and fixed $\mathrm{pH}_{\text {int }}=7$. The numerical values are shown in the Supporting Information. The energy values in the $y$ axis of part $\mathrm{c}$ are presented with an offset of $-3.497 \times 10^{6}$ for better visualization. 


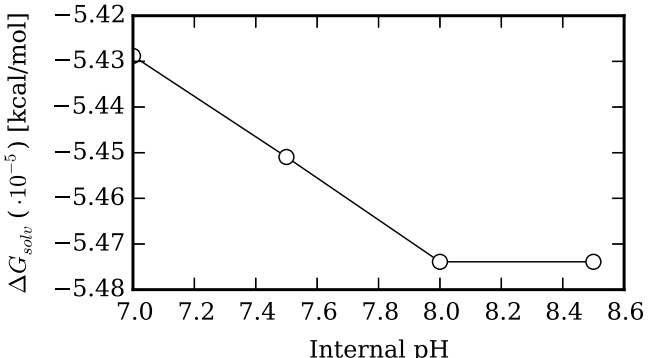

(a) Solvation energy

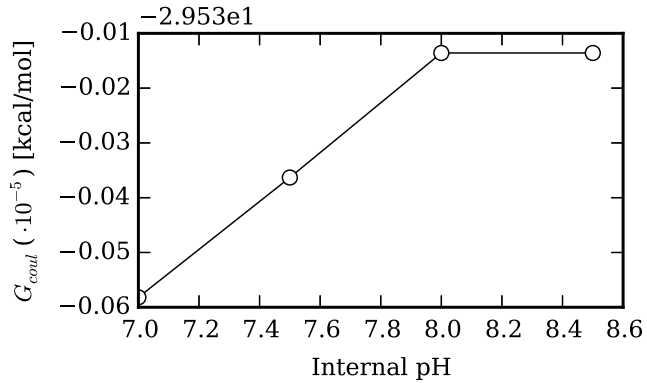

(b) Coulomb energy

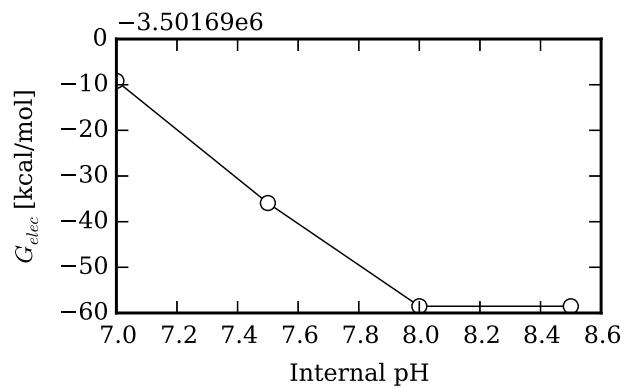

(c) Total electrostatic energy

Figure 4. Electrostatic free energy with varying internal $\mathrm{pH}$ and fixed $\mathrm{pH}_{\text {ext }}=8.5$. The numerical values are shown in the Supporting Information. The energy values in the $y$ axis of parts a and $c$ are presented with an offset of -29.53 and $3.502 \times 10^{6}$, respectively, for better visualization.

and 3; however, as we are interested in energy differences, our conclusions are not affected by this inaccuracy.

Table 2. Electrostatic Free Energy of the Capsid As It Disassembles by Placing 800 Negative Charges Inside the Capsid

\begin{tabular}{ccccc} 
& & \multicolumn{3}{c}{ energy $[\mathrm{kcal} / \mathrm{mol}]$} \\
\cline { 3 - 5 } stage no. & reference figure & solvation & Coulomb & total \\
1 & $2 \mathrm{a}$ & -547390 & -2954358 & -3501749 \\
2 & $2 \mathrm{~b}$ & -544237 & -2938631 & -3482868 \\
3 & $2 \mathrm{c}$ & -289386 & -3202676 & -3492062
\end{tabular}

Looking at the total electrostatic free energy in Figure $3 \mathrm{c}$ and Figure 4c, it becomes evident that the external $\mathrm{pH}$ has a much larger impact than the internal $\mathrm{pH}$ in the capsid stability. These figures show a drop in the free energy, however, the drop is far more important when the external $\mathrm{pH}$ increases. Moreover, in Figure 4c, the drop is only in the order of 60 $\mathrm{kcal} / \mathrm{mol}$, which is below the resolution of our numerical method.
Table 3. Electrostatic Free Energy of the Capsid As It Disassembles by Placing 1600 Negative Charges Inside the Capsid

\begin{tabular}{ccccc} 
& & \multicolumn{3}{c}{ energy $[\mathrm{kcal} / \mathrm{mol}]$} \\
\cline { 3 - 5 } stage no. & reference figure & solvation & Coulomb & total \\
1 & $2 \mathrm{a}$ & -547390 & -2954358 & -3501749 \\
2 & $2 \mathrm{~b}$ & -543040 & -2891099 & -3434139 \\
3 & $2 \mathrm{c}$ & -347984 & -3175389 & -3523373
\end{tabular}

Figures 3 and 4 also show that there is no clear relation between the free energy and the $\mathrm{pH}$ gradient across the capsid. For example, the free energy is higher for $\mathrm{pH}_{\text {int }}=7.0$ and $\mathrm{pH}_{\text {ext }}$ $=7.5$ compared to $\mathrm{pH}_{\text {int }}=8.0$ and $\mathrm{pH}_{\text {ext }}=8.5$, even though the $\mathrm{pH}$ difference is the same. This is due to the asymmetric importance of the internal and external $\mathrm{pH}$ on the free energy.

Moreover, it is interesting to analyze how the solvation and Coulomb terms contribute to the electrostatic free energy. Figure 3 and Figure 4 suggest that these energies tend to compensate, as the differences in solvation and Coulomb energies alone are large and opposite in sign, but the total free energy has a small change. In all our tests, however, the solvation energy changes prevail.

Energy Calculations of the Disassembling Process. According to Viso and co-workers, ${ }^{17}$ the alkaline $\mathrm{pH}$ inside the capsid deprotonates the genetic material, generating an electrostatic repulsive force that causes disassembling. Similar to that work, we model the deprotonated genetic material with negative point charges $\left(q=-1 q_{e}\right)$ placed randomly in a spherical shell region close to the capsid, as shown in Figure $2 \mathrm{~b}$, and compute the electrostatic free energy of the system. In these simulations, the shell region has no salt $(\kappa=0)$ and the dielectric constant of the solvent $(\epsilon=80)$.

Table 2 and Table 3 show the electrostatic contribution to the free energy in three different stages of the disassembling process:

1. Capsid assembled structure (PDB ID: $3 \mathrm{NAP}$ ) with $\mathrm{pH}=$ 8.5 everywhere, representing the capsid in an alkaline environment.

2. Capsid assembled structure with $\mathrm{pH}=8.5$ everywhere and negative charges in the spherical shell, to model the deprotonated genetic material.

3. Disassembled structure obtained from dynamic simulations with $\mathrm{Cl}^{-}$ions inside the capsid, and $\mathrm{pH}=8.5$ everywhere.

The results in Table 2 corresponds to simulations with 800 negative charges inside the capsid, whereas Table 3 presents calculations with 1600 charges.

The free energy changes from Table 2 and Table 3 are useful to study the stability of the system in the disassembling process. The jump from stage 1 to 2 is the energetic difference as the genetic material loses $\mathrm{H}^{+}$ions due to the alkaline environment. As expected, the free energy increases, indicating that the system is less stable. Looking at the contribution of the solvation and Coulombic parts of the free energy difference, this destabilization is mainly due to the Coulombic component, and it is greater in the case with 1600 ions.

The negative charges induce an electrostatic repulsion that pushes the capsid outward. In fact, comparing stages 2 and 3 in Table 2 and Table 3, the free energy drops, showing that the system is more stable as it disassembles, making it a possible disassembling trajectory. In this case, however, there is a 
compensation effect, as the solvation energy increases and the Coulomb term decreases. In absolute terms, the Coulomb contribution is larger, confirming that the electrostatic repulsion of the negatively charged genetic material leads the disassembling process. These observations agree with the mechanism proposed by Viso et al. ${ }^{17}$

\section{CONCLUSION}

The modeling of the large conformational changes associated with the viral capsid disassembly process via molecular simulations comprises real methodological and computational challenges. Here, we have presented a multiscale method that combines coarse-grained $\mathrm{MD}$ and $\mathrm{PB}$ models to simulate largescale viral capsids from a free energy perspective. More specifically, we studied the effect of $\mathrm{pH}$ and charge of the genetic material, and how free energy changes as the capsid disassembles. We noticed that the $\mathrm{pH}$ of the solvent has an important effect on stability, whereas in the internal region the impact is negligible. We also confirmed that as the genetic material loses protons, the free energy decreases as the capsid disassembles, showing that the trajectories computed with molecular simulations are indeed possible snapshots of the disassembly paths for the TrV. Our method for calculating free energies of viral and virus-like capsids presents a novel approach that complements the molecular understanding of the disassembly process of viruses. Part of this complementary information contributes with quantitative free energy landscapes of the disassembly process snapshots. In addition, we show that within a couple of hours a quantitative overview of the viral disassembly structures can be given. Thus, we present an agile method which is ready to be applied beyond proof-ofconcept systems. All around, we deliver a powerful computeraided tool for applied research. While interpreting experimental results is a challenging endeavor, our work can assist the advanced microscopy techniques which are currently developing towards image acquisition with video rates and thus reaching dynamical processes of viral capsids. As an outlook and aligned to experimental results, our pioneering method can be employed to tackle also phenomena like mechanicalcrowding in viral disassembly processes by combining molecular simulations to continuum mechanical models.

\section{ASSOCIATED CONTENT}

\section{S Supporting Information}

The Supporting Information is available free of charge at https://pubs.acs.org/doi/10.1021/acs.jcim.9b00883.

Influence of the $\mathrm{pH}$ gradient on the free energy and perspectives on the modeling of large conformational changes of viral capsid, (PDF)

Tcl file (ZIP)

\section{AUTHOR INFORMATION}

\section{Corresponding Authors}

*(C.D.C.) E-mail: christopher.cooper@usm.cl.

*(A.B.P.) E-mail: apoma@ippt.pan.pl.

*(H.V.G.) E-mail: horacio.guzman@ijs.si.

ORCID $\odot$

Christopher D. Cooper: 0000-0003-0282-8998

Adolfo B. Poma: 0000-0002-8875-3220

Horacio V. Guzman: 0000-0003-2564-3005

\section{Notes}

The authors declare no competing financial interest.

\section{ACKNOWLEDGMENTS}

The authors thank Matias Machado for providing us with initial configurations of the $\mathrm{TrV}$ capsids simulations and fruitful discussions regarding the SIRAH forcefield. In addition, the authors thank Torsten Stuehn, Tomasz Lipniacki and Simon Poblete for their feedback on the Manuscript. This research has been supported by the Slovenian Research Agency (Research Core Funding No. P1-0055); Deutsche Forschungsgemeinschaft (DFG, German Research Foundation), Project Number 233630050, TRR 146; the National Science Centre, Poland, under Grant No. 2017/26/D/NZ1/00466, and by CONICYT (Chile) through FONDECYT Iniciación No. 11160768 and Basal FB0821. The authors also thank the computer resources from CCTVal and MPCDF from the Max Planck Society.

\section{REFERENCES}

(1) Roos, W. H.; Gibbons, M. M.; Arkhipov, A.; Uetrecht, C.; Watts, N. R.; Wingfield, P. T.; Steven, A. C.; Heck, A. J. R.; Schulten, K.; Klug, W. S.; Wuite, G. J. L. Squeezing Protein Shells: how Continuum Elastic Models, Molecular Dynamics Simulations, and Experiments Coalesce at the Nanoscale. Biophys. J. 2010, 99, 1175-1181.

(2) Mateu, M. G. Mechanical Properties of Viruses Analyzed by Atomic Force Microscopy: a Virological Perspective. Virus Res. 2012, 168 (1-2), 1-22.

(3) Šiber, A.; Božič, A. L.; Podgornik, R. Energies and Pressures in Viruses: Contribution of Nonspecific Electrostatic Interactions. Phys. Chem. Chem. Phys. 2012, 14, 3746-3765.

(4) Zhao, G.; Perilla, J. R.; Yufenyuy, E. L.; Meng, X.; Chen, B.; Ning, J.; Ahn, J.; Gronenborn, A. M.; Schulten, K.; Aiken, C.; Zhang, P. Mature HIV-1 Capsid Structure by Cryo-electron Microscopy and All-atom Molecular Dynamics. Nature 2013, 497, 643-646.

(5) Llauró, A.; Guerra, P.; Kant, R.; Bothner, B.; Verdaguer, N.; De Pablo, P. J. Decrease in $\mathrm{pH}$ Destabilizes Individual Vault Nanocages by Weakening the Inter-protein Lateral Interaction. Sci. Rep. 2016, 6, 34143.

(6) Perilla, J. R.; Hadden, J. A.; Goh, B. C.; Mayne, C. G.; Schulten, K. All-Atom Molecular Dynamics of Virus Capsids as Drug Targets. J. Phys. Chem. Lett. 2016, 7, 1836-1844.

(7) Globisch, C.; Krishnamani, V.; Deserno, M.; Peter, C. Optimization of an Elastic Network Augmented Coarse Grained Model to Study CCMV Capsid Deformation. PLoS One 2013, 8, No. e60582.

(8) Wołek, K.; Cieplak, M. Self-assembly of Model Proteins into Virus Capsids. J. Phys.: Condens. Matter 2017, 29, 474003.

(9) Sun, J.; DuFort, C.; Daniel, M.-C.; Murali, A.; Chen, C.; Gopinath, K.; Stein, B.; De, M.; Rotello, V. M.; Holzenburg, A.; Kao, C. C.; Dragnea, B. Core-controlled Polymorphism in Virus-like Particles. Proc. Natl. Acad. Sci. U. S. A. 2007, 104, 1354-1359.

(10) Mashayak, S. Y.; Jochum, M. N.; Koschke, K.; Aluru, N. R.; Rühle, V.; Junghans, C. Relative Entropy and Optimization-Driven Coarse-Graining Methods in VOTCA. PLoS One 2015, 10, e0131754.

(11) Darré, L.; Machado, M. R.; Brandner, A. F.; González, H. C.; Ferreira, S.; Pantano, S. SIRAH: A Structurally Unbiased CoarseGrained Force Field for Proteins with Aqueous Solvation and LongRange Electrostatics. J. Chem. Theory Comput. 2015, 11, 723-739.

(12) Machado, M. R.; Barrera, E. E.; Klein, F.; Sóñora, M.; Silva, S.; Pantano, S. The SIRAH 2.0 Force Field: Altius, Fortius, Citius. J. Chem. Theory Comput. 2019, 15, 2719-2733.

(13) Kar, P.; Gopal, S. M.; Cheng, Y.-M.; Predeus, A.; Feig, M. PRIMO: a Transferable Coarse-grained Force Field for Proteins. J. Chem. Theory Comput. 2013, 9, 3769-3788.

(14) Monticelli, L.; Kandasamy, S. K.; Periole, X.; Larson, R. G.; Tieleman, D. P.; Marrink, S.-J. The MARTINI Coarse-grained Force 
Field: Extension to Proteins. J. Chem. Theory Comput. 2008, 4, 819834.

(15) Poma, A. B.; Cieplak, M.; Theodorakis, P. E. Combining the MARTINI and Structure-based Coarse-grained Approaches for the Molecular Dynamics Studies of Conformational Transitions in Proteins. J. Chem. Theory Comput. 2017, 13, 1366-1374.

(16) Ołdziej, S.; Czaplewski, C.; Liwo, A.; Chinchio, M.; Nanias, M.; Vila, J.; Khalili, M.; Arnautova, Y.; Jagielska, A.; Makowski, M.; Schafroth, H.; Kazmierkiewicz, R.; Ripoll, D.; Pillardy, J.; Saunders, J.; Kang, Y.; Gibson, K.; Scheraga, H. A. Physics-based Protein-structure Prediction Using a Hierarchical Protocol Based on the UNRES Force Field: Assessment in Two Blind Tests. Proc. Natl. Acad. Sci. U. S. A. 2005, 102, 7547-7552.

(17) Viso, J. F.; Belelli, P.; Machado, M.; González, H.; Pantano, S.; Amundarain, M. J.; Zamarreño, F.; Branda, M. M.; Guérin, D. M.; Costabel, M. D. Multiscale Modelization in a Small Virus: Mechanism of Proton Channeling and its Role in Triggering Capsid Disassembly. PLoS Comput. Biol. 2018, 14, No. e1006082.

(18) Guzman, H. V.; Tretyakov, N.; Kobayashi, H.; Fogarty, A. C.; Kreis, K.; Krajniak, J.; Junghans, C.; Kremer, K.; Stuehn, T. ESPResSo ++ 2.0: Advanced Methods for Multiscale Molecular Simulation. Comput. Phys. Commun. 2019, 238, 66-76.

(19) Poma, A. B.; Delle Site, L. Classical to Path-Integral Adaptive Resolution in Molecular Simulation: Towards a Smooth QuantumClassical Coupling. Phys. Rev. Lett. 2010, 104, 250201.

(20) Potestio, R.; Español, P.; Delgado-Buscalioni, R.; Everaers, R.; Kremer, K.; Donadio, D. Monte Carlo Adaptive Resolution Simulation of Multicomponent Molecular Liquids. Phys. Rev. Lett. 2013, 111, 060601.

(21) Praprotnik, M.; Kremer, K.; Delle Site, L. Adaptive Molecular Resolution via a Continuous Change of the Phase Space Dimensionality. Phys. Rev. E 2007, 75, 017701.

(22) Delgado-Buscalioni, R.; Kremer, K.; Praprotnik, M. Coupling Atomistic and Continuum Hydrodynamics through a Mesoscopic Model: Application to Liquid Water. J. Chem. Phys. 2009, 131, 244107.

(23) Kreis, K.; Kremer, K.; Potestio, R.; Tuckerman, M. E. From Classical to Quantum and Back: Hamiltonian Adaptive Resolution Path Integral, Ring Polymer, and Centroid Molecular Dynamics. J. Chem. Phys. 2017, 147, 244104.

(24) Zavadlav, J.; Melo, M. N.; Marrink, S. J.; Praprotnik, M. Adaptive Resolution Simulation of Polarizable Supramolecular Coarse-grained Water Models. J. Chem. Phys. 2015, 142, 244118.

(25) Squires, G., et al., PDB id: 3NAP, Triatoma Virus. 2013; https://www.rcsb.org/structure/3NAP.

(26) Control of Chagas Disease: Second Report of the World Health Organization Expert Committee; 2002.

(27) Machado, M. R.; González, H. C.; Pantano, S. MD Simulations of Viruslike Particles with Supra CG Solvation Affordable to Desktop Computers. J. Chem. Theory Comput. 2017, 13, 5106-5116.

(28) Abraham, M. J.; Murtola, T.; Schulz, R.; Páll, S.; Smith, J. C.; Hess, B.; Lindahl, E. GROMACS: High Performance Molecular Simulations through Multi-level Parallelism from Laptops to Supercomputers. SoftwareX 2015, 1-2, 19-25.

(29) Kutzner, C.; Páll, S.; Fechner, M.; Esztermann, A.; de Groot, B. L.; Grubmuller, H. More Bang for your Buck: Improved use of GPU nodes for GROMACS 2018. J. Comput. Chem. 2019, 40, 2418-2431.

(30) Machado, M. R.; Pantano, S. SIRAH Tools: Mapping, Backmapping and Visualization of Coarse-grained models. Bioinformatics 2016, 32, 1568-1570.

(31) See the Supporting Information.

(32) de Pablo, P. J. Atomic Force Microscopy of Virus Shells. Semin. Cell Dev. Biol. 2018, 73, 199-208.

(33) Guerrero, C. R.; Garcia, P. D.; Garcia, R. Subsurface Imaging of Cell Organelles by Force Microscopy. ACS Nano 2019, 13, 96299637.

(34) Martínez, M.; Cooper, C. D.; Poma, A. B.; Guzmán, H. V. Input Files for PyGBe to obtain results from "Free Energies of the Disassembly of
Viral Capsids from a Multiscale Molecular Simulation Approach 2019, DOI: 10.5281 /zenodo.3550043.

(35) Roux, B.; Simonson, T. Implicit Solvent Models. Biophys. Chem. 1999, 78, 1-20.

(36) Decherchi, S.; Masetti, M.; Vyalov, I.; Rocchia, W. Implicit Solvent Methods for Free Energy Estimation. Eur. J. Med. Chem. 2015, 91, 27-42.

(37) Levy, R. M.; Zhang, L. Y.; Gallicchio, E.; Felts, A. K. On the Nonpolar Hydration Free Energy of Proteins: Surface Area and Continuum Solvent Models for the Solute-Solvent Interaction Energy. J. Am. Chem. Soc. 2003, 125, 9523-9530.

(38) Cooper, C. D.; Bardhan, J. P.; Barba, L. A. A biomolecular Electrostatics Solver using Python, GPUs and Boundary Elements that can handle Solvent-filled Cavities and Stern Layers. Comput. Phys. Commun. 2014, 185, 720-729.

(39) Cooper, C. D.; Clementi, N. C.; Barba, L. A. Probing Protein Orientation near Charged Nanosurfaces for Simulation-assisted Biosensor Design. J. Chem. Phys. 2015, 143, 124709.

(40) Guzman, H. V.; Junghans, C.; Kremer, K.; Stuehn, T. Scalable and Fast Heterogeneous Molecular Simulation with Predictive Parallelization Schemes. Phys. Rev. E: Stat. Phys., Plasmas, Fluids, Relat. Interdiscip. Top. 2017, 96, 053311.

(41) Barnes, J.; Hut, P. A Hierarchical $O(N \log N)$ Force-Calculation Algorithm. Nature 1986, 324, 446-449.

(42) Li, P.; Johnston, H.; Krasny, R. A Cartesian Treecode for Screened Coulomb Interactions. J. Comput. Phys. 2009, 228, 38583868.

(43) Cooper, C. D.; Clementi, N. C.; Forsyth, G.; Barba, L. A. PyGBe: Python, GPUs and Boundary Elements for Electrostatics. https://github.com/pygbe/pygbe.

(44) Wagoner, J.; Baker, N. A. Solvation Forces on Biomolecular Structures: A Comparison of Explicit Solvent and PoissonBoltzmann Models. J. Comput. Chem. 2004, 25, 1623-1629.

(45) Fogolari, F.; Brigo, A.; Molinari, H. The Poisson-Boltzmann Equation for Biomolecular Electrostatics: a Tool for Structural Biology. J. Mol. Recognit. 2002, 15, 377-392.

(46) Bardhan, J. P. Biomolecular Electrostatics - I Want your Solvation (Model). Comput. Sci. Discovery 2012, 5, 013001.

(47) Decherchi, S.; Rocchia, W. A General and Robust Ray-castingbased Algorithm for Triangulating Surfaces at the Nanoscale. PLoS One 2013, 8, No. e59744.

(48) van der Schoot, P.; Zandi, R. Impact of the Topology of Viral RNAs on their Encapsulation by Virus Coat Proteins. J. Biol. Phys. 2013, 39, 289-299.

(49) Erdemci-Tandogan, G.; Wagner, J.; van der Schoot, P.; Podgornik, R.; Zandi, R. Effects of RNA Branching on the Electrostatic Stabilization of Viruses. Phys. Rev. E: Stat. Phys., Plasmas, Fluids, Relat. Interdiscip. Top. 2016, 94, 022408.

(50) Smrek, J.; Kremer, K. Small Activity Differences Drive Phase Separation in Active-Passive Polymer Mixtures. Phys. Rev. Lett. 2017, 118, 098002.

(51) Dolinsky, T. J.; Nielsen, J. E.; McCammon, J. A.; Baker, N. A. PDB2PQR: an Automated Pipeline for the Setup of PoissonBoltzmann Electrostatics Calculations. Nucleic Acids Res. 2004, 32, W665-W667.

(52) Valsson, O.; Parrinello, M. Variational Approach to Enhanced Sampling and Free Energy Calculations. Phys. Rev. Lett. 2014, 113, 090601.

(53) Perego, C.; Valsson, O.; Parrinello, M. Chemical Potential Calculations in Non-Homogeneous Liquids. J. Chem. Phys. 2018, 149, 072305.

(54) Maragliano, L.; Vanden-Eijnden, E. A Temperature Accelerated Method for Sampling Free Energy and Determining Reaction Pathways in Rare Events Simulations. Chem. Phys. Lett. 2006, 426, 168-175.

(55) Agirre, J.; Goret, G.; LeGoff, M.; Sanchez-Eugenia, R.; Marti, G. A.; Navaza, J.; Gurin, D. M. A.; Neumann, E. Cryo-electron Microscopy Reconstructions of Triatoma Virus Particles: a Clue to 
Unravel Genome Delivery and Capsid Disassembly. J. Gen. Virol. 2013, 94, 1058-1068.

(56) Snijder, J.; Uetrecht, C.; Rose, R. J.; et al. Probing the Biophysical Interplay Between a Viral Genome and its Capsid. Nat. Chem. 2013, 5, 502-509.

(57) Steppert, P.; Burgstaller, D.; Klausberger, M.; Tover, A.; Berger, E.; Jungbauer, A. Quantification and Characterization of Virus-like Particles by Size-exclusion Chromatography and Nanoparticle

Tracking Analysis. J. Chromatogr. A 2017, 1487, 89-99. 